\title{
A Passenger Flow Risk Forecasting Algorithm for High-Speed Railway Transport Hub Based on Surveillance Sensor Networks
}

\author{
Zhengyu Xie $^{1,2}$ and Yong Qin ${ }^{2}$ \\ ${ }^{1}$ School of Traffic and Transportation, Beijing Jiaotong University, Beijing 100044, China \\ ${ }^{2}$ State Key Laboratory of Rail Traffic Control and Safety, Beijing Jiaotong University, Beijing 100044, China \\ Correspondence should be addressed to Yong Qin; yqin@bjtu.edu.cn
}

Received 18 March 2016; Revised 14 May 2016; Accepted 7 June 2016

Academic Editor: Iftikhar Ahmad

Copyright (C) 2016 Z. Xie and Y. Qin. This is an open access article distributed under the Creative Commons Attribution License, which permits unrestricted use, distribution, and reproduction in any medium, provided the original work is properly cited.

Passenger flow risk forecasting is a vital task for safety management in high-speed railway transport hub. In this paper, we considered the passenger flow risk forecasting problem in high-speed railway transport hub. Based on the surveillance sensor networks, a passenger flow risk forecasting algorithm was developed based on spatial correlation. Computational results showed that the proposed forecasting approach was effective and significant for the high-speed railway transport hub.

\section{Introduction}

In the 12th Five-Year Plan (2011-2015) period, high-speed railway in China had a fast development. As of December 31, 2015, the high-speed railway lines amounting to a total length of $19000 \mathrm{~km}$ were operated in mainland China, and most cities with more than 50 million populations were covered by the high-speed railway transport networks. With the fast development of high-speed railway, high-speed railway transport hub has become a vital node of passenger transport networks and several transport modes, that is, civil aviation, highway, urban rail transit, and public transport transferred in high-speed railway transport hub. Massive passenger flow collecting and distributing brings several potential risks to the safety management of high-speed railway transport hub.

At present, most of high-speed railway transport hubs have emergency plans for different risks, especially passenger flow risk. When risk values meet the threshold, these plans are activated immediately. But current approach only passively responded for emergency and lacks actively detection and forecasting of risks. With wide application of intelligent video surveillance in high-speed railway transport hubs, a comprehensive surveillance sensor networks is gradually formed, which provides powerful supports for risk detecting and forecasting. Based on real-time passenger flow status obtained by surveillance sensor networks, passenger flow risk forecasting can effectively prevent risks, reduce risks value, and decrease the negative effects caused by risks. So it is necessary for high-speed railway transport hubs to study on passenger flow risk forecasting approach based on surveillance sensor networks.

The rest of this paper is organized as follows: Section 2 reviews the relevant literature. The passenger flow risk forecasting frame is introduced in Section 3, and Section 4 proposes a passenger flow risk forecasting algorithm based on spatial correlation. Computational experiments are conducted in Section 5 and finally Section 6 covers the conclusion.

\section{Literature Review}

Currently, video surveillance is the key approach to detect the potential risks in transport hubs. Several passenger image processing and intelligent detecting algorithms were developed for rapid and accurate detection of passenger flow status [1-8]. A real-time system for detecting and tracking multiple people when they appear in a group was proposed. Several computational models, that is, a silhouettebased shape model, a motion model, and correlation-based matching methods were employed to track multiple people before, during, and after occlusion [1]. For automatically 
tracking pedestrian, a pedestrian tracking system was proposed, consisting of three subsystems, which calculates the image processing, object tracking, and traffic flow variables. The first subsystem performs image processing analysis while the second subsystem carries out the tracking of pedestrians by matching the features and tracing the pedestrian numbers frame by frame [2]. In order to accurately and promptly detect potential safety hazard, a modified background model based on Dempster-Shafer theory and a passenger flow status recognition algorithm based on features of image connected domain were proposed to improve the accuracy and realtime performance of passenger flow detection [3]. To improve detection accuracy in terms of feature extraction, a novel feature of gradient self-similarity (GSS) was present, which was computed from HOG and was applied to capture the patterns of pairwise similarities of local gradient patches [4]. For the challenging of abrupt illumination, occlusions, out of field of view, and cluttered backgrounds, a novel tracking framework was developed, which consists of two steps: image shadow removal and tracking by association [5]. A sparse representation based approach is proposed for pedestrian detection from thermal images. The approach first adopted the histogram of sparse code to represent image features and then detect pedestrian with the extracted features in a unimodal and a multimodal framework, respectively [6]. In order to accurately detect potential safety hazard hidden in passenger flow, a hybrid forecasting approach was proposed to obtain the passenger flow status [7]. By thoroughly analyzing and optimizing each step of the detection pipeline, a pedestrian detection system was proposed based on deep learning, adapting a general-purpose convolutional network to the task at hand [8].

Some studies focused on causing and handling of the potential risks [9-15]. A pedestrian evacuation simulation model based on the extended cellular automata was proposed with the consideration of heterogeneous behavioral tendencies in humans, and the model was applied to optimize buildings to reduce evacuation times [9]. A more comprehensive conceptual model was introduced for the representation of the impact of fatigue on the performance of evacuees during building stair evacuation. The model was presented considering its conceptual formulation and the issues associated with its implementation [10]. A multigrid model was proposed to simulate evacuation with guiders, and the effects of guider type, guider number, guider distribution, and guidance strategy on evacuation were discussed [11]. The selfish and selfless behaviors were considered as two main factors in evacuation, and selfishness- and selflessnessbased model of pedestrian room evacuation was proposed [12]. A new multiagent based congestion evacuation model incorporating panic behavior was proposed for simulating pedestrian evacuation in public places such as a stadium. Pedestrians in this model were divided into four classes and each pedestrian's status can be either normal, overtaken, or casualty [13]. The evacuation process from a room without visibility was investigated by both experiment and modeling. Some typical characteristics of blind evacuation, including the preference of choosing left-hand side direction and following behavior, were found from the experiment
[14]. A pedestrian evacuation modeling framework was developed, which used volunteered geographical information from OpenStreetMap and simplified queuing-network model to estimate evacuation time, detect bottlenecks, and test different evacuation strategies [15].

According to the literature review above, current studies only focused on the potential risks detection and risk handling. For passenger flow forecasting, most of studies concerned passenger flow demand, arriving, and departure volume forecasting [16-19]. Specific literature on risk forecasting is scarce. The risk forecasting is an important connection between potential risks detection and risk handling. Based on the detection result, forecasting the passenger flow risk can well master the variation trend of risk and adopt suitable handling operations. So in this paper, we consider the passenger flow risk forecasting in high-speed railway transport hub. Based on surveillance sensor networks, a spatial correlation forecasting algorithm is developed to forecast the passenger flow risk of bottleneck areas.

\section{Passenger Flow Risk Forecasting Frame}

In this section, the passenger flow risk forecasting frame is described in three aspects. Firstly, surveillance sensor networks of high-speed railway transport hub are introduced. Secondly, forecasting mechanism is described. Based on the previous two parts, the forecasting procedure is designed in the last part.

3.1. Surveillance Sensor Networks of High-Speed Railway Transport Hub. According to the different surveillance purposes and focuses, the sensors in surveillance sensor networks of high-speed railway transport hub can be classified into three types [20], key area monitoring sensors, passenger line monitoring sensors, and complete coverage monitoring sensors, which are shown in Figure 1.

For the three types of sensors (Figure 1), our study mainly focuses on the passenger flow risk forecasting of key area. The forecasting not only use the passenger flow data detected by key area monitoring sensors but also used the passenger flow data obtained by passenger line monitoring sensors.

3.2. Passenger Flow Risk Forecasting Mechanism. The accuracy of passenger flow risk forecasting in key area is influenced by several factors, that is, current risk status of key area, risk status of correlated surveillance areas in passenger line, and service capability of equipment in key area. So it is hard to obtain an accurate risk forecasting value by only using the risk status of key area.

In this paper, we consider the passenger flow risk forecasting of key area under the surveillance sensor networks in high-speed railway transport hub. By analyzing the relationship between key area and correlated surveillance areas in passenger line, a forecasting algorithm is developed based on spatial correlation.

The spatial correlation between key area and correlated areas in passenger line can be defined as the impact on key area risk caused by correlated areas. A sample of spatial 


\begin{tabular}{|c|c|c|}
\hline \multirow{3}{*}{$\begin{array}{l}\text { Sensor } \\
\text { in } \\
\text { surveillance } \\
\text { sensor } \\
\text { networks }\end{array}$} & $\begin{array}{l}\text { Key area } \\
\text { monitoring } \\
\text { sensors }\end{array}$ & $\begin{array}{l}\text { (i) Sensors in different key areas are independent and do not have any relevance } \\
\text { (ii) Sensors do not need adjustment after setting } \\
\text { (iii) Sensors have front-end event detecting software }\end{array}$ \\
\hline & $\begin{array}{c}\text { Passenger line } \\
\text { monitoring sensors }\end{array}$ & $\begin{array}{l}\text { (i) Sensors should be set following the passenger line } \\
\text { (ii) Sensors in the same passenger line have association } \\
\text { (iii) Sensors do not need adjustment after setting } \\
\text { (iv) The data acquired by sensors should be continuously transferred to the control } \\
\text { center to process }\end{array}$ \\
\hline & $\begin{array}{l}\text { Complete coverage } \\
\text { monitoring sensors }\end{array}$ & $\begin{array}{l}\text { (i) Sensors should cover all the function areas in HRTH } \\
\text { (ii) The monitoring areas of sensors should reduce overlaps as much as possible } \\
\text { (iii) Sensors can adjust monitoring areas after setting } \\
\text { (iv) The data acquired by sensors should be continuously transferred to the control } \\
\text { center to be stored }\end{array}$ \\
\hline
\end{tabular}

FIGURE 1: Sensors in surveillance sensor networks.

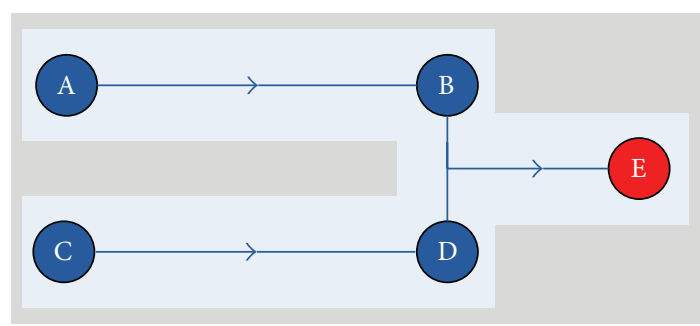

FIGURE 2: A sample of spatial correlation.

correlation is shown in Figure 2. In the figure, the red point is the key area, blue points are correlated surveillance areas, and the blue arrows are passenger line. The passengers moved from $\mathrm{A}$ to $\mathrm{B}$ and $\mathrm{C}$ to $\mathrm{D}$ and converge to $\mathrm{E}$. The $\mathrm{A}, \mathrm{B}, \mathrm{C}$, and $\mathrm{D}$ have spatial correlation with $\mathrm{E}$, and the spatial correlation degrees of $\mathrm{B}$ and $\mathrm{D}$ are larger than the spatial correlation degrees of $\mathrm{A}$ and $\mathrm{C}$.

3.3. Passenger Flow Risk Forecasting Procedure. In the surveillance sensor networks of high-speed railway transport hub, based on the spatial correlation, there are three main steps in the passenger flow risk forecasting procedure, which are shown in Figure 3.

Step 1. Calculate the risk value $r(x)$ of correlated surveillance areas by using the passenger flow status of the areas.

Step 2. Calculate spatial correlation degrees $s(x, y)$ between key area and correlated surveillance areas based on the surveillance sensor networks of high-speed railway transport hub.

Step 3. Forecast the risk value $R(e)$ of key area based on the current risk value $r(e)$ of key area, $r(x)$, and $s(x, y)$ obtained by Steps 1 and 2 .

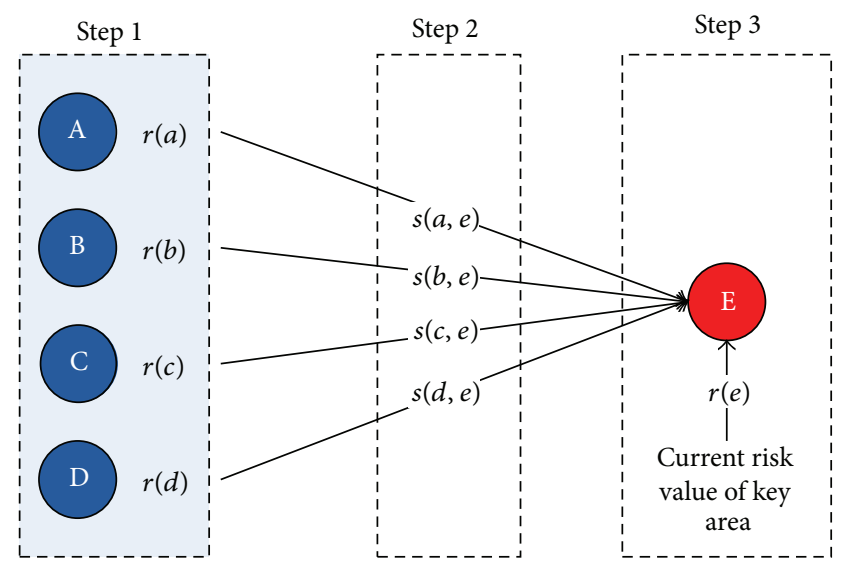

FIgURE 3: Main steps in the passenger flow risk forecasting procedure.

\section{A Passenger Flow Risk Forecasting Algorithm Based on Spatial Correlation}

According to passenger flow risk forecasting procedure mentioned in Section 3, the calculation method of passenger flow risk value and forecasting algorithm based on spatial correlation will be discussed in this section.

4.1. Passenger Flow Risk Value Calculation. In this paper, we adopt passenger flow amount to calculate the risk value in key area and correlated surveillance areas. And three types of passenger flow status parameters are used, which are current passenger flow amount of area $A_{1}$, average passenger flow amount of area in statistic period $A_{2}$, and max passenger flow amount of area in statistic period $A_{3}$. Based on plenty of data experiments for different areas in high-speed railway transport hubs, the passenger flow risk values in key area and correlated surveillance areas can be calculated as follows. 
(1) While current passenger flow amount is less than or equal to average passenger flow amount in statistic period, $A_{1} \leq A_{2}$,

$$
r(x)=3-10\left(\frac{A_{2}-A_{1}}{A_{2}}\right)
$$

(2) While current passenger flow amount is larger than average passenger flow amount in statistic period and less than or equal to max passenger flow amount of area in statistic period, $A_{2}<A_{1} \leq A_{3}$,

$$
r(x)=\frac{4 A_{1}+3 A_{3}-7 A_{2}}{A_{3}-A_{2}} .
$$

(3) While current passenger flow amount is larger than max passenger flow amount of area in statistic period, $A_{1}>$ $A_{3}$,

$$
r(x)=7+10\left(\frac{A_{1}-A_{3}}{A_{3}}\right)
$$

4.2. Spatial Correlation Degree Calculation. The spatial correlation reflects the risk correlation between key area and correlated surveillance areas. And the spatial correlation degree is an important indicator to represent the impact on passenger flow risk of key area caused by the risk value of correlated surveillance areas.

$r_{i}=\left\{r_{i}(t) \mid t=1,2, \ldots, n\right\}$ is a time series set, representing risk values of one key area and $i$ correlated surveillance areas at different time. $r_{0}(t)$ is the risk values of key area in $t$ period, and $r_{i}(t)$ is the risk values of the $i$ th correlated surveillance area in $t$ period. The spatial correlation degree between the key area and ith correlated surveillance area in $t$ period can be calculated as follows:

$$
s\left(r_{0}(t), r_{i}(t)\right)=\frac{m+\omega M}{\Delta(0, i)+\omega M},
$$

where $\Delta(0, i)=\left|r_{0}(t)-r_{i}(t)\right|, m=\min _{i} \min _{t} \Delta(0, i), M=$ $\max _{i} \max _{t} \Delta(0, i)$, and $\omega$ is the identification coefficient, $\omega \in$ $(0,1)$.

According to the incidence extent, each spatial correlation between key area and one correlated surveillance area has a correlation weight coefficient, which can be calculated by

$$
\alpha_{i}=\frac{s\left(r_{0}(t), r_{i}(t)\right)}{\sum_{j=1}^{n} s\left(r_{0}(t), r_{j}(t)\right)} .
$$

4.3. Forecasting Algorithm Based on Spatial Correlation. For a key area $k$, it has $n$ correlated surveillance areas $c k_{i}, i=$ $1,2, \ldots, n$. Based on the passenger flow risk value of $k$ and $c k_{i}$ and the spatial correlation among their areas, the initial passenger flow risk of $k$ in $t$ period can be forecasted as follows:

$$
r_{k}^{\prime}(t)=\sum_{i=1}^{n} \alpha_{i} r_{i}\left(t-t_{i}\right)
$$

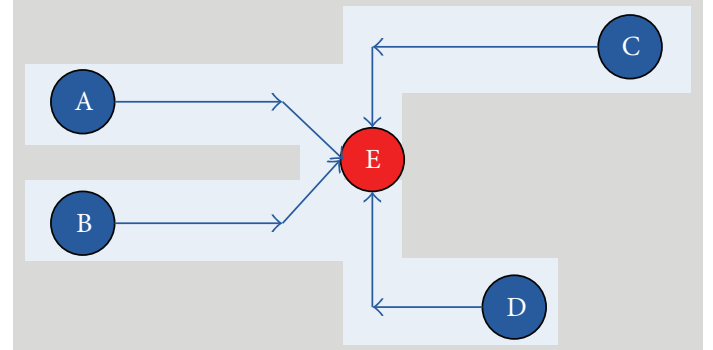

FIGURE 4: Schematic representation of areas.

where $\alpha_{i}$ is the correlation weight coefficient between $k$ and $c k_{i}$ and $t_{i}$ is the correlation time between $k$ and $c k_{i}$.

In actual forecasting, for improving the accuracy, we employ the initial forecasting value and actual passenger flow risk values before $t$ period to forecast the passenger flow risk of $k$ in $t$ period. The forecasting algorithm is shown as follows:

$$
\begin{aligned}
r_{k}(t) & =r_{k}(t-1)+\Delta r_{k}(t) \\
& =r_{k}(t-2)+r_{k}(t-1)+\Delta r_{k}(t) \\
& =r_{k}(0)+\Delta r_{k}(1)+\Delta r_{k}(2)+\cdots+\Delta r_{k}(t) .
\end{aligned}
$$

Set $\Delta r_{k}(t)=\Delta r_{k}^{\prime}(t)$, and $\Delta r_{k}^{\prime}(t)=r_{k}^{\prime}(t)-r_{k}^{\prime}(t-1)$. So the forecasting algorithm can be transferred as follows:

$$
\begin{aligned}
r_{k}(t) & =r_{k}^{\prime}(t)-r_{k}^{\prime}(0)+r_{k}(0) \\
& =\sum_{i=1}^{n} \alpha_{i} r_{i}\left(t-t_{i}\right)-\sum_{i=1}^{n} \alpha_{i} r_{i}(0)+r_{k}(0),
\end{aligned}
$$

where $r_{k}(0)$ is the actual passenger flow risk value of $k$ at the initial forecasting period and $r_{i}(0)$ is the actual passenger flow risk value of $c k_{i}$ at the initial forecasting period.

\section{Computational Experiments}

In this section, computational experiments are conducted to verify passenger flow risk algorithm proposed above. A specific key area in high-speed railway transport hub is taken as an example. This area is an escalator connecting waiting hall and entrances. The schematic representation of their areas is shown in Figure 4.

The $\mathrm{E}$ is the escalator. $\mathrm{A}$ and $\mathrm{B}$ are two metro transfer points; the passengers arriving by metro enter the hub from these two points. $\mathrm{C}$ is an entrance; the passengers arriving by public transport enter the hub from this entrance. D is a park entrance; the passengers arriving by private car enter the hub from this entrance. Both of these areas have intelligent surveillance, which can automatically detect the passenger flow amount and calculate the risk value. Based on the real detection data from intelligent surveillance sensors, the forecasting algorithm developed in Section 3 is employed to forecast the passenger flow risk of area E. We set 10 minutes as a forecasting period, and firstly experiment on 6 hours $(12: 00 \mathrm{am}-6: 00 \mathrm{pm})$ is conducted. The computational result 


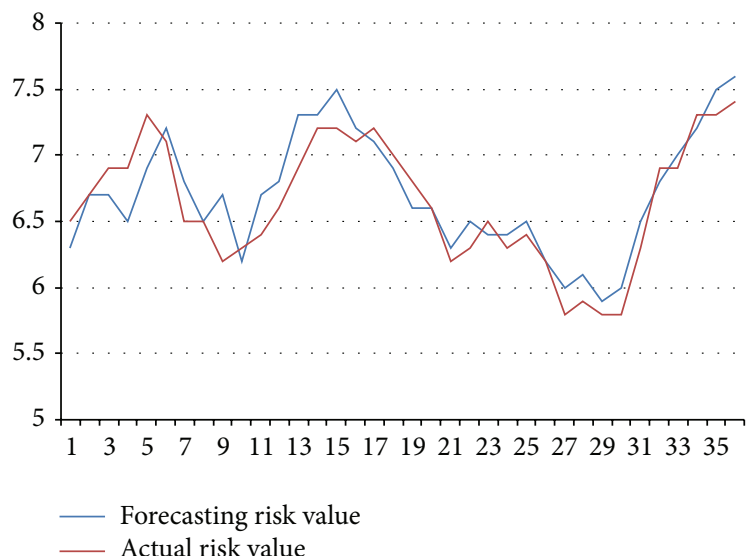

FIGURE 5: Comparison between forecasting risk value and actual risk value.

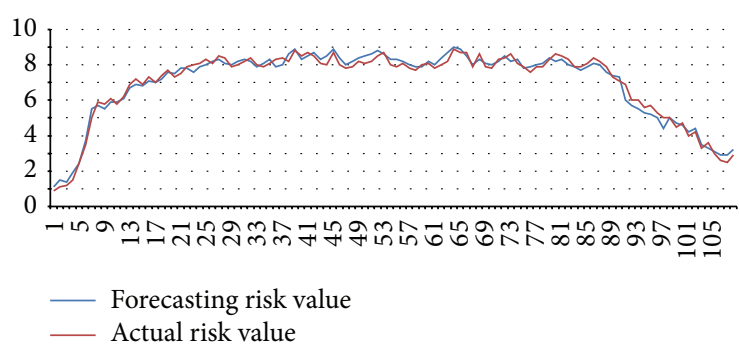

FIGURE 6: Computational result of a festival day.

is shown in Table 1, and comparison between forecasting risk value and actual risk value is shown in Figure 5.

As observed in Figure 5, the forecasting risk values obtained by our approach are close to actual risk value; the average gap between forecasting risk value and actual risk value is $2.6 \%$. And the variation trend of passenger flow risk is well forecasted by our approach. For illustrating the performance of our forecasting approach facing mass passenger flow, a festival day $(6: 00 \mathrm{am}-12: 00 \mathrm{pm})$ is selected as an example. The computational result is shown in Figures 6 and 7.

As observed in Figures 6 and 7, our forecasting approach has a good performance for mass passenger flow in festival days. It can well forecast variation trend of passenger flow risk in a whole day time (6:00 am-12:00 pm is a normal operation time of hub) except some low risk periods. Generally, our forecasting approach can meet the demands of safety management in high-speed railway transport hub.

\section{Conclusion}

In this paper, we considered the passenger flow risk forecasting problem in high-speed railway transport hub. According to the surveillance sensor network of hub, a spatial correlation degree calculation between the key area and the correlated surveillance area was proposed, and a passenger flow risk forecasting algorithm based on spatial correlation was developed. Computational experiments on a key area in
TABLE 1: Computational result of 6 hours (12:00 am-6:00 pm).

\begin{tabular}{|c|c|c|c|c|}
\hline Hour & Period & $\begin{array}{c}\text { Forecasting } \\
\text { risk value }\end{array}$ & $\begin{array}{c}\text { Actual risk } \\
\text { value }\end{array}$ & Gap \\
\hline \multirow{6}{*}{$12: 00 \mathrm{am}$} & 1 & 6.3 & 6.5 & $3.08 \%$ \\
\hline & 2 & 6.7 & 6.7 & $0.00 \%$ \\
\hline & 3 & 6.7 & 6.9 & $2.90 \%$ \\
\hline & 4 & 6.5 & 6.9 & $5.80 \%$ \\
\hline & 5 & 6.9 & 7.3 & $5.48 \%$ \\
\hline & 6 & 7.2 & 7.1 & $1.41 \%$ \\
\hline \multirow{6}{*}{$1: 00 \mathrm{pm}$} & 7 & 6.8 & 6.5 & $4.62 \%$ \\
\hline & 8 & 6.5 & 6.5 & $0.00 \%$ \\
\hline & 9 & 6.7 & 6.2 & $8.06 \%$ \\
\hline & 10 & 6.2 & 6.3 & $1.59 \%$ \\
\hline & 11 & 6.7 & 6.4 & $4.69 \%$ \\
\hline & 12 & 6.8 & 6.6 & $3.03 \%$ \\
\hline \multirow{6}{*}{ 2:00 pm } & 13 & 7.3 & 6.9 & $5.80 \%$ \\
\hline & 14 & 7.3 & 7.2 & $1.39 \%$ \\
\hline & 15 & 7.5 & 7.2 & $4.17 \%$ \\
\hline & 16 & 7.2 & 7.1 & $1.41 \%$ \\
\hline & 17 & 7.1 & 7.2 & $1.39 \%$ \\
\hline & 18 & 6.9 & 7.0 & $1.43 \%$ \\
\hline \multirow{6}{*}{$3: 00 \mathrm{pm}$} & 19 & 6.6 & 6.8 & $2.94 \%$ \\
\hline & 20 & 6.6 & 6.6 & $0.00 \%$ \\
\hline & 21 & 6.3 & 6.2 & $1.61 \%$ \\
\hline & 22 & 6.5 & 6.3 & $3.17 \%$ \\
\hline & 23 & 6.4 & 6.5 & $1.54 \%$ \\
\hline & 24 & 6.4 & 6.3 & $1.59 \%$ \\
\hline \multirow{6}{*}{ 4:00 pm } & 25 & 6.5 & 6.4 & $1.56 \%$ \\
\hline & 26 & 6.2 & 6.2 & $0.00 \%$ \\
\hline & 27 & 6.0 & 5.8 & $3.45 \%$ \\
\hline & 28 & 6.1 & 5.9 & $3.39 \%$ \\
\hline & 29 & 5.9 & 5.8 & $1.72 \%$ \\
\hline & 30 & 6.0 & 5.8 & $3.45 \%$ \\
\hline \multirow{6}{*}{ 5:00 pm } & 31 & 6.5 & 6.3 & $3.17 \%$ \\
\hline & 32 & 6.8 & 6.9 & $1.45 \%$ \\
\hline & 33 & 7.0 & 6.9 & $1.45 \%$ \\
\hline & 34 & 7.2 & 7.3 & $1.37 \%$ \\
\hline & 35 & 7.5 & 7.3 & $2.74 \%$ \\
\hline & 36 & 7.6 & 7.4 & $2.70 \%$ \\
\hline
\end{tabular}

high-speed railway transport hub showed that the proposed forecasting approach is effective to forecast the passenger flow risk of key area in hub. In future, considering several key areas risk, forecasting the whole safety status of high-speed railway transport hub is a possibility for further research.

\section{Competing Interests}

The authors declare that there is no conflict of interests regarding the publication of this paper. 


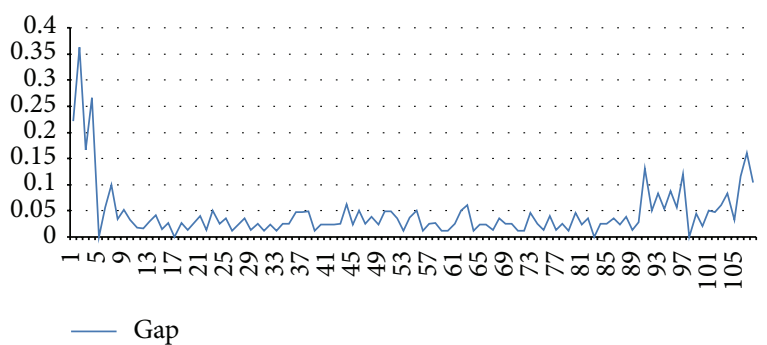

FIGURE 7: Gap between forecasting risk value and actual risk value in a festival day.

\section{Acknowledgments}

This research was supported by the Fundamental Research Funds for the Central Universities (Grant no. 2015JBM044) and the Talented Faculty Funds of Beijing Jiaotong University (Grant no. 2014RC005).

\section{References}

[1] I. Haritaoglu, D. Harwood, and L. S. Davis, "Hydra: multiple people detection and tracking using silhouettes," in Proceedings of the 10th International Conference on Image Analysis and Processing (ICIAP '99), pp. 280-285, IEEE, Venice, Italy, September 1999.

[2] K. Teknomo, Y. Takeyama, and H. Inamura, "Tracking system to automate data collection of microscopic pedestrian traffic flow," in Proceedings of the 4th Eastern Asia Society for Transportation Studies, vol. 3, no. 1, pp. 11-25, Hanoi, Vietnam, October 2001.

[3] S. Wu, R. Laganière, and P. Payeur, "Improving pedestrian detection with selective gradient self-similarity feature," Pattern Recognition, vol. 48, no. 8, pp. 2364-2376, 2015.

[4] Z. Y. Xie, L. M. Jia, Y. Qin, and L. Wang, "Passenger flow detection of video surveillance: a case study of high-speed railway transport hub in China," Elektronika ir Elektrotechnika, vol. 21, no. 1, pp. 48-53, 2015.

[5] X. Shen, X. Sui, K. Pan, and Y. Tao, "Adaptive pedestrian tracking via patch-based features and spatial-temporal similarity measurement," Pattern Recognition, vol. 53, pp. 163-173, 2016.

[6] B. Qi, V. John, Z. Liu, and S. Mita, "Pedestrian detection from thermal images: a sparse representation based approach," Infrared Physics \& Technology, vol. 76, pp. 157-167, 2016.

[7] Z. Y. Xie, L. M. Jia, Y. Qin, and L. Wang, "A hybrid temporalspatio forecasting approach for passenger flow status in Chinese high-speed railway transport hub," Discrete Dynamics in Nature and Society, vol. 2013, Article ID 239039, 7 pages, 2013.

[8] D. Tomè, F. Monti, L. Baroffio, L. Bondi, M. Tagliasacchi, and S. Tubaro, "Deep convolutional neural networks for pedestrian detection," http://arxiv.org/abs/1510.03608.

[9] D. Li and B. Han, "Behavioral effect on pedestrian evacuation simulation using cellular automata," Safety Science, vol. 80, pp. 41-55, 2015.

[10] E. Ronchi, P. A. Reneke, and R. D. Peacock, "A conceptual fatigue-motivation model to represent pedestrian movement during stair evacuation," Applied Mathematical Modelling, vol. 40, no. 7-8, pp. 4380-4396, 2016.

[11] S. Cao, W. Song, and W. Lv, "Modeling pedestrian evacuation with guiders based on a multi-grid model," Physics Letters,
Section A: General, Atomic and Solid State Physics, vol. 380, no. 4, pp. 540-547, 2016.

[12] X. Song, L. Ma, Y. Ma, C. Yang, and H. Ji, "Selfishnessand Selflessness-based models of pedestrian room evacuation," Physica A: Statistical Mechanics and Its Applications, vol. 447, pp. 455-466, 2016.

[13] J. Wang, L. Zhang, Q. Shi, P. Yang, and X. Hu, "Modeling and simulating for congestion pedestrian evacuation with panic," Physica A: Statistical Mechanics and Its Applications, vol. 428, pp. 396-409, 2015.

[14] S. Cao, W. Song, W. Lv, and Z. Fang, "A multi-grid model for pedestrian evacuation in a room without visibility," Physica A: Statistical Mechanics and Its Applications, vol. 436, pp. 45-61, 2015.

[15] B. Kunwar, F. Simini, and A. Johansson, "Large scale pedestrian evacuation modeling framework using volunteered geographical information," Transportation Research Procedia, vol. 2, pp. 813-818, 2014.

[16] Z. Ma, J. Xing, M. Mesbah, and L. Ferreira, "Predicting shortterm bus passenger demand using a pattern hybrid approach," Transportation Research Part C: Emerging Technologies, vol. 39, pp. 148-163, 2014.

[17] C. Zhou, P. Dai, F. Wang, and Z. Zhang, "Predicting the passenger demand on bus services for mobile users," Pervasive and Mobile Computing, vol. 25, pp. 48-66, 2016.

[18] G. Xie, S. Wang, and K. K. Lai, "Short-term forecasting of air passenger by using hybrid seasonal decomposition and least squares support vector regression approaches," Journal of Air Transport Management, vol. 37, pp. 20-26, 2014.

[19] Y. Wei and M.-C. Chen, "Forecasting the short-term metro passenger flow with empirical mode decomposition and neural networks," Transportation Research Part C: Emerging Technologies, vol. 21, no. 1, pp. 148-162, 2012.

[20] Z. Y. Xie and Y. Qin, "Sensor networks hierarchical optimization model for security monitoring in high-speed railway transport hub," Journal of Sensors, vol. 2015, Article ID 951242, 9 pages, 2015. 


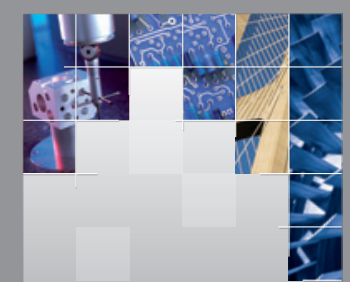

\section{Enfincering}
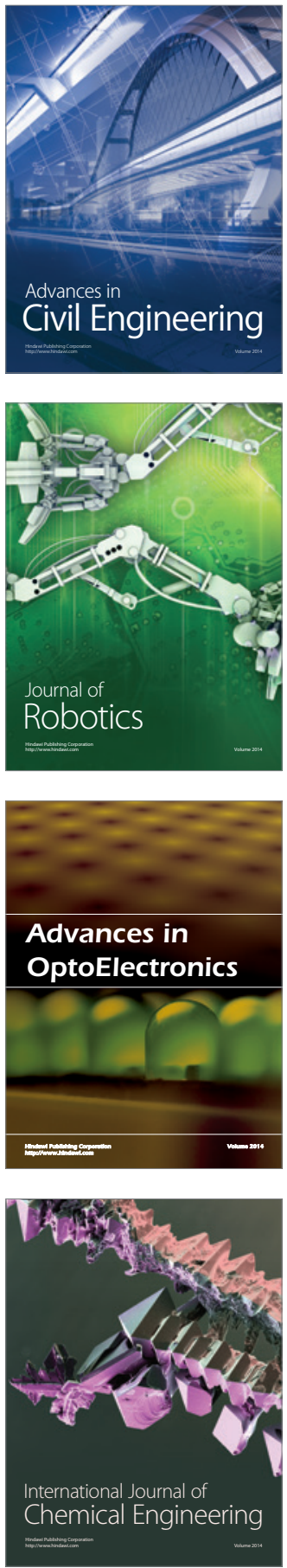

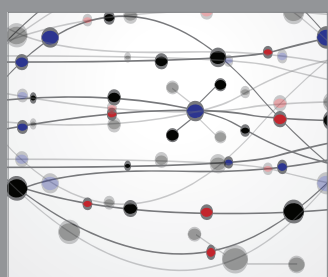

The Scientific World Journal

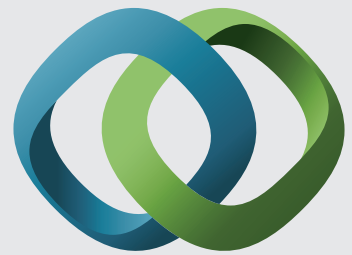

\section{Hindawi}

Submit your manuscripts at

http://www.hindawi.com
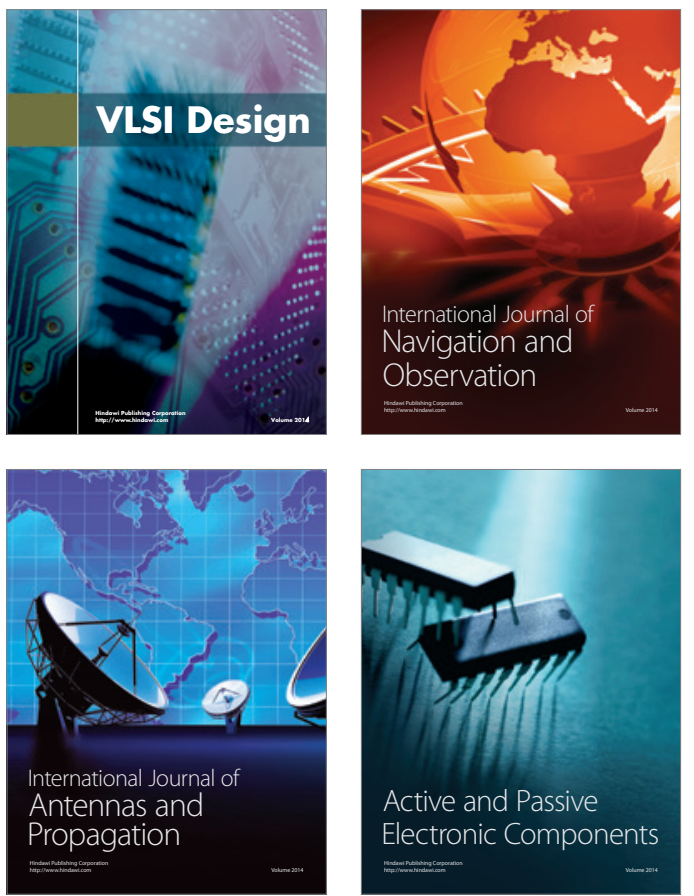
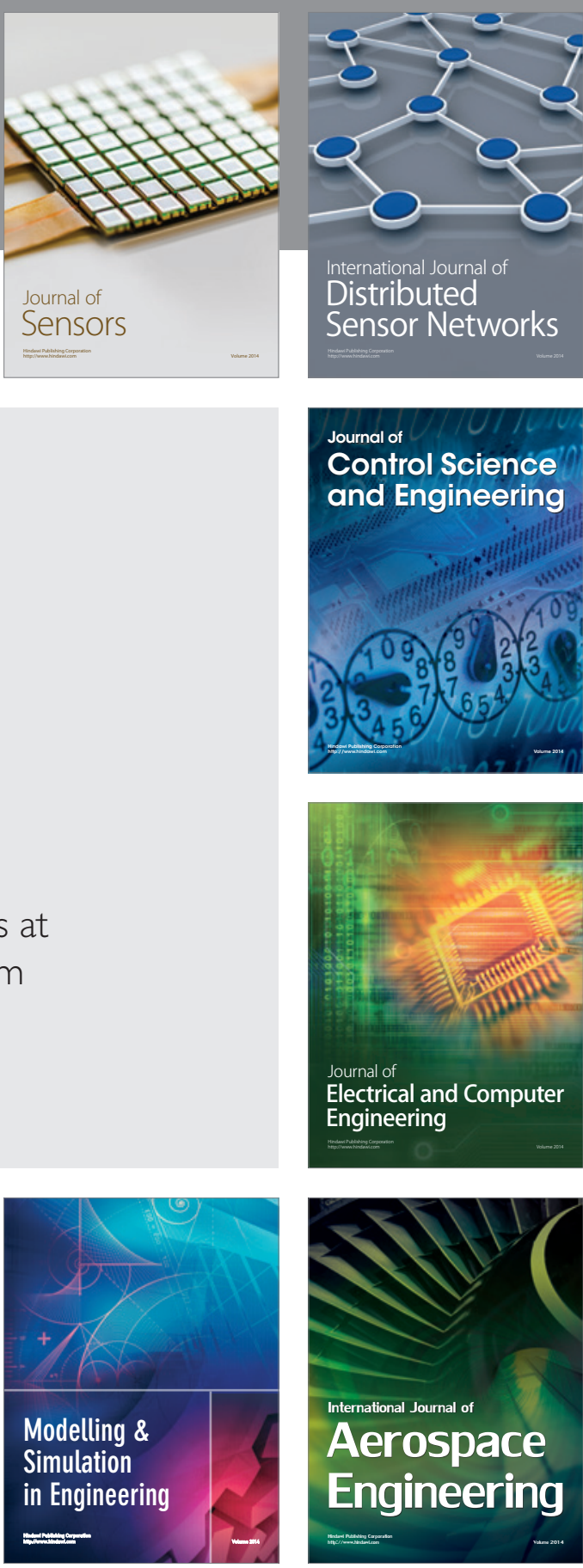

International Journal of

Distributed

Sensor Networks

Journal of

Control Science

and Engineering
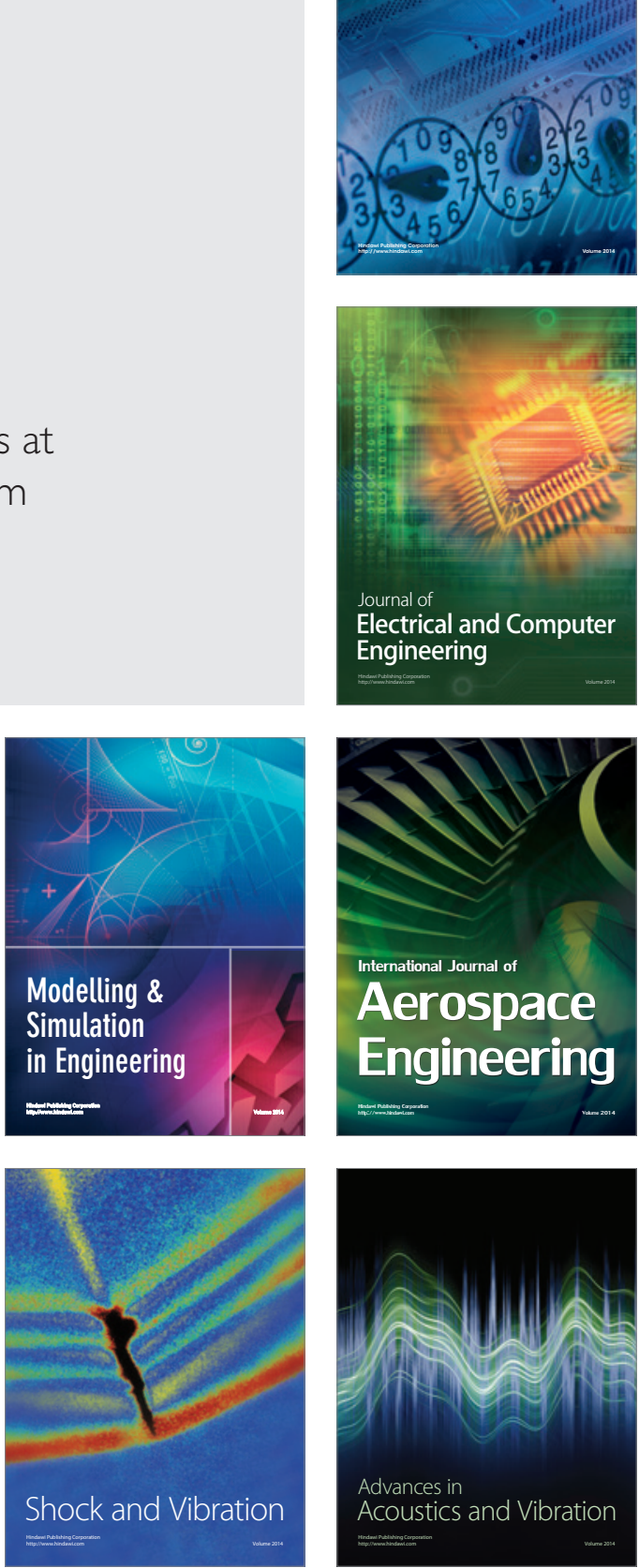